\title{
Erratum zu: Chemische Kreisläufe in der Natur
}

\section{Erratum zu: \\ V. Hopp, Chemische Kreisläufe in der Natur, https://doi.org/10.1007/978-3-662-55860-7}

Auf den folgenden Seiten im oben genannten Buch waren einige Fehler vorhanden.

In Kapitel II auf Seite 41 wurde die Einheit „,m“ in der zweiten Formel irrtümlicherweise unten statt oben gesetzt. Also haben wir die Formel in „,kg·m“ geändert, inhaltlich wurde das Kapitel jedoch nicht verändert.

In Kapitel XXVIII auf Seite 898 unter der Abbildung 17 wurde bei den Erläuterun-

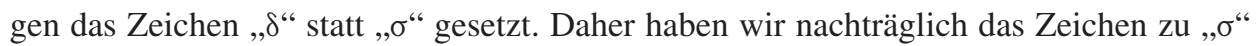
geändert, inhaltlich wurde das Kapitel jedoch nicht verändert.

Im Anhang (Kapitel XXIX) auf Seite 915 wurde die Einheit „N“ an zwei Stellen fälschlicherweise mit einem Kleinbuchstaben statt einem Großbuchstaben abgebildet. Daher haben wir nachträglich die Einheit in einen Großbuchstaben geändert, inhaltlich wurde das Kapitel jedoch nicht verändert.

Im Anhang (Kapitel XXIX) auf Seite 916 wurde die Einheit „N“ in der ersten Zeile fälschlicherweise mit einem Kleinbuchstaben statt einem Großbuchstaben abgebildet. Daher haben wir nachträglich die Einheit in einen Großbuchstaben geändert, inhaltlich wurde das Kapitel jedoch nicht verändert.

Die korrigierten Versionen der Kapitel sind verfügbar unter https://doi.org/10.1007/978-3-662-55860-7_2

https://doi.org/10.1007/978-3-662-55860-7_28

https://doi.org/10.1007/978-3-662-55860-7_29 